\title{
CT Imaging Anatomy and Application in the Head and Neck
}

\author{
Shaoyin Duan ${ }^{1,}$, Simin Lin $^{1}$ and Feng Ye ${ }^{1}$ \\ ${ }^{1}$ Medical Imaging Department, Zhongshan Hospital of Xiamen University, Xiamen 361004, China \\ axmdsy@xmzsh.com
}

Keywords: Head and neck; Tomography; X-ray computed; Image processing; Computer assisted

\begin{abstract}
By comparing the R-VR, This paper will evaluate the SFOF-VR imaging applied in the head and neck. Based on the original scanning data, SFOF-VR and R-VR were performed on 35 subjects (including the normal, with variations and/or lesions) from the head-neck CTA examination. Comparisons were made between the image features of SFOF-VR and R-VR in showing the anatomy, variation and lesion in the head and neck. SFOF-VR showed independently or jointly the $\mathrm{C} 0, \mathrm{C} 1, \mathrm{C} 2, \mathrm{VA}, \mathrm{VVP}$ and their lesions or abnormality with different opacity and colors. In the 35 subjects, there are 6 normal subjects, 16 with variations $(7$ cases in VA and 9 in AAJ) and 13 with lesions (6 cases with VA arteriosclerosis and 7 with fracture or dislocation in AAJ. Comparisons showed that images of SFOF-VR are more clear and comprehensive than those of $\mathrm{R}-\mathrm{VR}$ in displaying the normal anatomy and abnormal changes $(\mathrm{P}<0.05)$. SFOF-VR can overcome the R-VR's shortcoming and more clearly show the lesions without overlapping than the $\mathrm{R}-\mathrm{VR}$ for imaging diagnosis and surgical treatment.

(Supplemental abbreviations: SFOF-VR = separating, fusing, opacifying and false-coloring volume rendering; $\mathrm{R}-\mathrm{VR}=$ routine volume rendering; $\mathrm{VA}=$ vertebral artery; $3 \mathrm{D}=$ three-dimensional; AFDLAJ = articulating facet displacement of lateral atlantoaxial joint; occipital $=\mathrm{C} 0$; atlas $=\mathrm{C} 1$; epistropheus $=\mathrm{C} 2$; venous vascular plexus $=\mathrm{VVP}$ )
\end{abstract}

\section{Introduction}

CT 3D-imaging with SFOF-VR is a post-process imaging technique with CT scanning volume-data. It can form 3D-images of single-structure up to 8, then merge these images and change opacity or pseudo-color respectively, finally get the satisfactory 3D-images with the characteristic of clearly and directly showing the anatomic structure or lesions. The AAJ is located in the deep head-neck boundary area, where there are many important blood vessels and nerves with complicated relation which is difficult to be observed with regional anatomy or two-dimensional image [1]. At the present, 3D-imaging of R-VR has been widely applied in clinical diagnosis, anatomy teaching and operation simulation [2], while SFOF-VR has not been reported in detail. This study is to discuss the SFOF-VR technique and application in the head and neck.

\section{Material and Methods}

General Information. In our hospital, SFOF-VR imaging of 35 subjects, selected from the head-neck CTA examination during May 1st 2008 and May 31st 2009 (20 males, 15 females, aged from 29 to 76 years, mean age 52.3 \pm 2.5 ), were retrospectively performed. There are 6 normal cases, 29 with variations or/and lesions on atlantoaxial joint or VA.

Equipment and Technique. 64-multi-detector spiral CT scanner, together with Advantage Workstation 4.2 (Light Speed VCT, GE Company, USA) was used. Contrast medium (Omnipaque, $300 \mathrm{mgI} / \mathrm{ml})$ with the total amount of $1.5 \mathrm{ml} \sim 2.0 \mathrm{ml} / \mathrm{kg}$ was injected in velocity of $3.0 \mathrm{ml} / \mathrm{s} \sim 4.0$ $\mathrm{ml} / \mathrm{s}$ with the pressure injector (Stellant, Medrad Co., USA) in this study.

Scanning Method. 25s and 45s after the contrast medium was injected into the forearm vein, 2 phases scans were performed with the slice thickness of $0.625 \mathrm{~mm}$, pitch of 0.984 . Scanning ranges is from the aortic arch to the top of skull. The scanning parameters include tube voltage of $120 \mathrm{Kv}$, tube current of $250 \mathrm{~mA}$, pitch of 1.375 , slice thickness of $0.625 \mathrm{~mm}$. Images standard reconstruction is with slice thickness of $0.625 \mathrm{~mm}$, increment of $0.3 \mathrm{~mm}$. 
Image Processing. On the Advantage Workstation 4.2, first the R-VR was performed, then SFOF-VR. On the R-VR interface, the function of "Auto-select" was used to select the "Clear Destination", "Add Structure" and "VA Color" to form and false-color the VA, occipital, atlas, epistropheus and venous vascular plexus respectively, then used the "Save/Recall" to save them temporarily with the names of S01, S02, S03 … Finally used the "Merge View" to merge these images of the S01, S02, S03 $\cdots$ from the "Save/Recall", then to adjust the opacity or fasle - color for obtaining clearly 3D-images and saved them to observe and measure the lesions or structures.

Image Evaluation and Criterion. Obtained the 3D-images with the SFOF-VR, radiologists (at least 3 professors or associate professors of radiology with more than 10 years of experience on the 3D CTA diagnosis) make the images evaluation. The abilities of $3 \mathrm{D}$ images in showing the structures or lesions were rated as excellent $(++++$, shown the bone or vessels without any structure effect), good (+++, shown the bone or vessels with a little other structure effect), passable (++, shown then bone or vessels with some other structure artifacts), poor (+, shown the bone or vessels with many other structure artifacts) and negative (-, not shown the bone or vessels). Statistical comparisons were made with Fisher exact probability test between R-VR and SFOF-VR on showing the anatomic structure, variation or foci and target structure.

\section{Results}

SFOF-VR Image and Technique. 3D images of SFOF-VR were obtained with the scanning and imaging parameters. Anatomic structures were clearly and directly shown, and the variations or lesions were observed with any rotation. Technique of separating can only show one single structure, such as the $\mathrm{C} 0, \mathrm{C} 1, \mathrm{C} 2$, VA or VVP. That of fusing can form the automatic superposition of multi-structures on a 3D-image. That of opacifying can retain or hide one or some structures to display the target Structure as you like. That of false-coloring can attach different color on different structures (VA attaches the color of red, VVP of blue, C1 of yellow and C2 of green) to show them for differentiating each other, while R-VR did not (Fig. 1).

Normal Anatomy, Variation and Diseases Diagnosis. In the total 35 cases, 6 normal subjects were shown on the SFOF-VR 3D images, which independently or jointly displayed the C0, C1, C2, VA and VVP, and avoided affecting each other or being sheltered. The whole courses of VA, going through the transverse foramen of $\mathrm{C} 1-\mathrm{C} 2$, were clearly revealed. 16 cases were found with variations, of which 7 cases was found variations in VA (abnormal course in 3, multi-branches 2 and fenestrated 2), 6 congenital arcuate foramen of atlas (unilateral in 4, bilateral in 2) and 3 congenital defects of posterior arch of atlas. 13 cases with lesion were diagnosed, including the atlantoaxial subluxation and/or fractures in 7 cases, vertebrobasilar atherosclerosis in 5 and VA aneurysm in 1. Showing the foci with SFOF-VR was in good agreement with that of DSA or surgery.

Comparison of 3D-Images. Comparisons were made in the image features or processing between SFOF-VR and R-VR (table1). There are the significant difference between SFOF-VR and $\mathrm{R}-\mathrm{VR}$ in showing the anatomic structure, variation or foci and target structure (Table $2, \mathrm{P}<0.001$ ).

Table 1 Comparison of SFOF-VR and R-VR 3D-image in showing anatomy and foci of bone and blood vessel at the craniocervial junction

\begin{tabular}{|c|c|c|c|c|c|c|c|}
\hline $\begin{array}{l}\text { Showing the } \\
\text { structures and } \\
\text { image features }\end{array}$ & $\begin{array}{l}\text { Anantmic } \\
\text { Structure }\end{array}$ & $\begin{array}{c}\text { Variation } \\
\text { and foci }\end{array}$ & $\begin{array}{c}\text { Selecting } \\
\text { incision }\end{array}$ & $\begin{array}{c}\text { Selecting } \\
\text { remain/color }\end{array}$ & $\begin{array}{c}\text { target } \\
\text { Structure }\end{array}$ & $\begin{array}{l}\text { non-target } \\
\text { structure }\end{array}$ & $\begin{array}{c}\text { Image } \\
\text { edge }\end{array}$ \\
\hline SFOF-VR & ++++ & ++++ & ++++ & ++++ & ++++ & - & ++ \\
\hline R-VR & ++ & ++ & - & - & ++ & ++ & ++ \\
\hline
\end{tabular}


Table 2 Statistical comparisons of 35 cases related data about the $3 \mathrm{~d}$-images between SFOF-VR and R-VR

\begin{tabular}{|c|c|c|c|c|c|c|c|}
\hline \multirow{2}{*}{ Showing content } & \multirow{2}{*}{ 3D-imaging } & \multicolumn{5}{|c|}{ Rated images } & \multirow{2}{*}{$P$ value* } \\
\hline & & excellent & good & passable & poor & negative & \\
\hline \multirow[t]{2}{*}{ Anatomic structure } & SFOF-VR & 25 & 7 & 3 & 0 & 0 & \multirow{2}{*}{$1.48 \mathrm{E}-11$} \\
\hline & $\mathrm{R}-\mathrm{VR}$ & 2 & 5 & 24 & 4 & 0 & \\
\hline \multirow{2}{*}{ Variation and foci } & SFOF-VR & 23 & 7 & 5 & 0 & 0 & \multirow{2}{*}{$3.94 \mathrm{E}-07$} \\
\hline & R-VR & 5 & 6 & 23 & 1 & 0 & \\
\hline \multirow{2}{*}{ Target Structure } & SFOF-VR & 26 & 3 & 6 & 0 & 0 & \multirow{2}{*}{ 2.65E-11 } \\
\hline & R-VR & 0 & 7 & 25 & 3 & 0 & \\
\hline
\end{tabular}

*form the Fisher exact probability test.

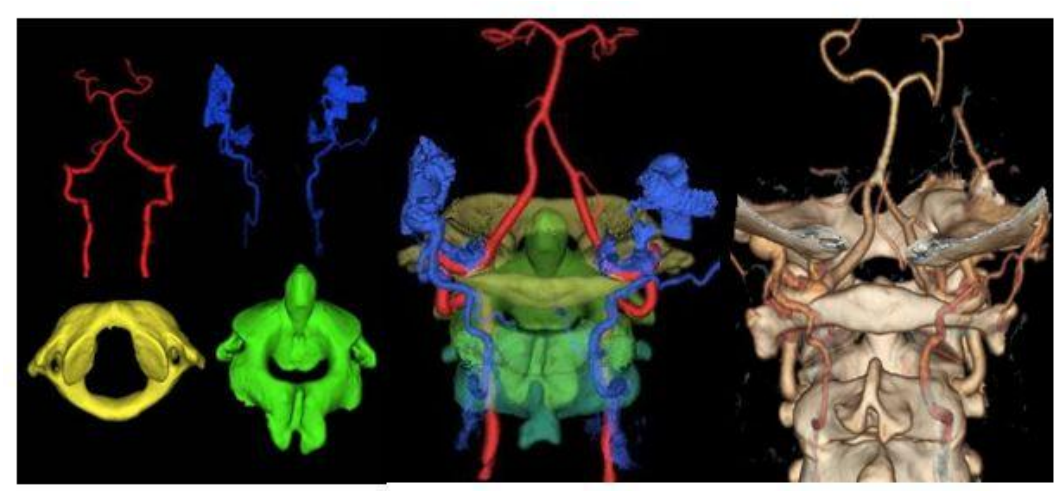

Figure 1. SFOF-VR 3D

imaging independently or jointly shows the VVP with the blue, $\mathrm{C} 1$ with the yellow and $\mathrm{C} 2$ with the green, while R-VR 3D imaging shows the overlapped structures of craniocervical junction (Fig. 1).

\section{Discussion}

Technical Features. SFOF-VR is a new imaging method in showing the anatomic structures with the separating, fusing, opacifying and false-coloring technique. It can overcome the shortcomings of $\mathrm{R}-\mathrm{VR}$ and achieved clearer the imaging vivisection. At the same time, SFOF-VR can provide more anatomic information for clinical diagnosis and surgery treatment. SFOF-VR's separating technique can be used to draw out one of the object structure from the craniocervical region to show it without any overlap or shelter. Its fusing technique can be used to superpose the multi-object structures to show them and observe their relations. The opacifying can be used to gain the "perspective ability" by clearing the shelter to show the deep structure. The false-coloring can be used to map various colors into the gray-scale images to improve the ability of people's eyes in differentiating the object. All of these techniques were combined, there are many advantages in displaying the complex anatomy and their relations [3].

Comparisons with R-VR. SFOF-VR can show different structures in different color, for example, the vertebral artery in red color, atlas in yellow and epistropheus in green. It is easy for doctors to observe VA going along the atlas or epistropheus and going through the transverse foramen or congenital arcuate foramen. 3D-images of SFOF-VR can display the whole course of vertebral artery by clearing the atlas and epistropheus or show the atlas, epistropheus with clearing the other structures. It shows the VA or its atheroma or stenosis as DSA does, and the atlas, epistropheus as bone specimen. With the selective cutting function, SFOF-VR 3D-imaging can improve the ability of differentiating anatomical structures and observing their lesions. Of course, SFOF-VR compared with R-VR, there are some shortcomings, for example, time-consuming 
relatively, creditability affected by operator's professional standards and without showing the non-target structures, whose lesions may not be shown $[4,5]$.

Clinical Applications and Prospects. SFOF-VR can display the single or multi-structures with different color and opacity on a 3D-image. It can avoid shelters to observe their size, shape and relations. With the technical development and improvement, imaging vivisection with SFOF-VR can be easily and have good application, including the anatomical quantitative analysis, medical basic and clinical application, of which is helpful in showing mini abnormality and giving correct imaging diagnosis, especially for complicated anatomic variations and tiny or covered lesions [6-8]. SFOF-VR can accurately locate the lesion and clearly show their relations, and thus provides more anatomic information for selecting optimal surgical path or treatment method. Furthermore, the combination of the technology and finite element modeling will have great effect on the study of regional anatomy, biomechanical analysis and surgical design $[9,10]$.

\section{Acknowledgments}

We would like to express our thanks for the support of National Natural Science Foundation of China (81071214) and Natural Science Foundation of Fujian Province, China (Grand No. 2015J01535).

\section{References}

[1] S.Y. Duan, F. Ye, and J.H. Kang. Three-dimensional CT study on normal anatomical features of atlanto-axial joints, Surg Radiol Anat. 29 (2007) 83-88.

[2] M.Yamazaki, A. Okawa, M. Hashimoto, A. Aiba, Y. Someya and M. Koda. Abnormal course of the vertebral artery at the craniovertebral junction in patients with Down at the craniovertebral junction in patients with Down, Neuroradiology. 50 (2008) 485-490.

[3] K. Radcliff, C. Kepler, C. Reitman, J. Harrop and A. Vaccaro. CT and MRI-based diagnosis of craniocervical dislocations: the role of the occipitoatlantal ligament, Clin Orthop Relat Res. 470(2012):1602-13.

[4] S.Y. Duan, S.M. Lv, F. Ye and Q.C. Lin. Imaging anatomy and variation of vertebral artery and bone structure at craniocervical junction, Eur Spine Journal. 18 (2009) 1102-1108.

[5] T.V. Le , E. Dakwar, S. Hann, E. Effio , A.A. Baaj, C. Martinez, F.L. Vale and J.S. Uribe. Computed tomography-based morphometric analysis of the human occipital condyle for occipital condyle-cervical fusion, J Neurosurg Spine. 15 (2011) 328-331.

[6] S. Mohan, M. Agarwal and B. Pukenas. Computed Tomography Angiography of the Neurovascular Circulation, Radiol Clin North Am. 54(2016) 147-162.

[7] A. Leone, A. Costantini, M Visocchi, A. Vestito, P. Colelli, N. Magarelli, C. Colosimo and L. Bonomo. The role of imaging in the pre- and postoperative evaluation of posterior occipito-cervical fusion, Radiol Med. 117(2012) 636-653.

[8] Y.W. Chen, J.H. Yen, W.H. Chen, I.C. Chen, C.S. Lai, C.T. Lu. And D.Y. Song. Preoperative Computed Tomography Angiography for Evaluation of Feasibility of Free Flaps in Difficult Reconstruction of Head and Neck, Ann Plast Surg.76 (2016) S19-24.

[9] W. Ji, G.G. Kong, M.H. Zheng, X.Y. Wang, J.T. Chen and Q.A. Zhu. Computed tomographic morphometric analysis of pediatric clival screw placement at the craniovertebral junction, Spine (Phila Pa 1976). 40 (2015) 259-365.

[10]O.Maillot, A. Attyé, E. Boyer, O. Heck, A. Kastler, S. Grand, S. Schmerber and A.Krainik. Post traumatic deafness: a pictorial review of $\mathrm{CT}$ and MRI findings. Insights Imaging. 7(2016):341-350. 